\title{
Highly Ionized Gas as a Diagnostic of the Inner NLR
}

\author{
M. J. Ward ${ }^{1}$, J. Mullaney ${ }^{1}$, C. Jin ${ }^{1}$, and R. Davies ${ }^{2}$ \\ ${ }^{1}$ Department of Physics, Durham University, Durham, UK \\ ${ }^{2}$ Max-Planck Institut für extraterrestrische Physik, Garching, Germany
}

\begin{abstract}
The spectra of AGN, from the ultraviolet to the near infrared, exhibit emission lines covering a wide range of ionization states, from neutral species such as [O I] $\lambda 6300$, up to $[\mathrm{Fe}$ IV] $\lambda 5303$. Here we report on some recent studies of the properties of highly ionized lines (HILs), plus two case studies of individual objects. Future IFU observations at high spatial and good spectral resolution will probe the excitation and kinematics of the gas in the zone between the extended NLR and unresolved BLR. Multi-component SED fitting can be used to link the source of photoionization with the strengths and ratios of the HILs.
\end{abstract}

Keywords. galaxies: active, galaxies: kinematics and dynamics, galaxies: nuclei, galaxies: Seyfert

\section{Introduction}

The detection and initial investigations of highly ionized emission-line species in AGN has been possible for several decades (e.g., Pelat et al. 1981; Penston et al. 1984). However it is only relatively recently that sensitive near-IR spectroscopy and IFUs have renewed the impetus to use these features as diagnostics of both the ionization processes and gas kinematics. At high energies, i.e., X-rays and the ultraviolet, observational evidence has been accumulating in support of the presence of outflows. Although estimates of the mass in the outflow are difficult to make and assumption-dependent, in some cases it may be substantial, and the associated kinetic energy a significant fraction of the bolometric luminosity (Reeves et al. 2009).

\section{Samples of AGN with Highly Ionized Lines}

Thanks to advances in the technology of near-IR arrays, we now have significant numbers of AGN with high-quality spectra from 1-2.5 $\mu \mathrm{m}$ (e.g., Riffel et al. 2006; Landt et al. 2008), although generally the near-IR spectral resolution is not yet comparable to that of similar samples at optical wavelengths. Based mostly on optical studies, there is now convincing evidence of blueshifts of the highly ionized species with respect to the systemic velocity, as shown by, for example, Rodriguez-Ardila et al. (2006) and Mullaney \& Ward (2008). In a recent study, Gelbord et al. (2009) used spectra mined from the SDSS catalog to select 63 AGN based on the strength of their [Fex] $\lambda 6374$ line emission. Clearly such a sample has strong selection effects, but it is valuable to compare its properties with other studies which almost aways draw their targets from the optically brighest and best-observed AGN. Gelbord et al. (2009) find strong correlations between the X-ray continuum and lines of [Fex] and [FeXI], as well as a trend for the broadest HIL profiles to have the highest blueshifts.

Smaller samples of AGN have been observed via narrow-band imaging in the emission line of [Si IV] $\lambda 2.48 \mu \mathrm{m}$. Prieto et al. (2005) showed that, in a small sample of Seyfert $2 \mathrm{~s}$, the region is extended from a few tens up to $150 \mathrm{pc}$, making it significantly less extended 


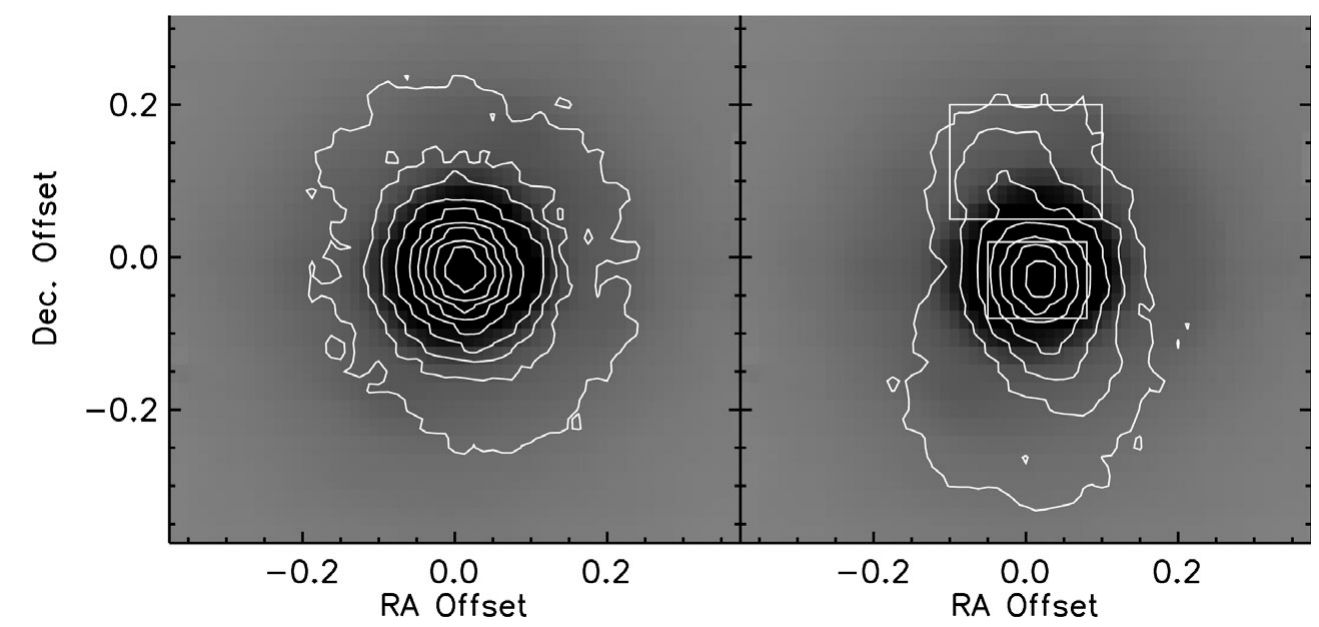

Figure 1. NGC 3783, SINFONI data. Left: contours of broad Br $\gamma$ flux assumed to be unresolved. Right: Contour of [Si VI] emission line at $1.963 \mu \mathrm{m}$ (Mullaney et al., in preparation).

than the NLR. Furthermore, several studies of the HIL, for example recent work by Mullaney \& Ward (2008), have shown that the 44 profiles of the HILs can often be deconvolved into several components, and that the broadest of these has a FWHM in between those of the BLR and the NLR (as typified by the [O III] $\lambda 5007$ line). Finally, a very recent study of HILs using HST+STIS data (Mazzalay et al. 2010) confirms substantial blueshifts and also detects line splitting at the core of some galaxies.

\section{Case Studies}

Although statistical studies of high-ionization line species detected in large samples of AGN, such as those mentioned above, are very useful, these need to be complemented by detailed studies of some individual cases. In their seminal study of the optical and near infrared coronal lines in NGC 1068, Marconi et al. (1996) demonstrated the diagnostic potential of combining several ratios of HIL of various elements and ionization species. However, NGC 1068 is a Seyfert 2, and presumably some proportion of the region emitting the highly ionized gas will be blocked by the torus. It would therefore be desirable to make detailed studies of Seyfert 1s.

\subsection{Akn 564}

Mullaney et al. (2009) used new spectroscopic data on the NLS1 Akn 564 and extended the modeling techniques described by Ferguson et al. (1997). They were able to build a self-consistent model that explains both the line ratios and kinematics of the highly ionized gas. In essence, this model invokes gas liberated from the the dusty torus and then accelerated to its terminal velocity. In principle, this model can be tested and constrained using additional HIL species and high spatial-resolution spectroscopy.

\section{2. $N G C 3783$}

NGC 3783 is a classic type 1 Seyfert and, due to its proximity and high flux, it has been intensively studied at all energies. We have used recent near-IR data from SINFONI to extract maps of several emission lines in the $K$-band. Of particular interest in this context is the map of [Si VI] at rest wavelength $1.963 \mu \mathrm{m}$ (Figure 1). An elongation of a few tenths of an arcsec can be seen towards the north (top). The projected physical 


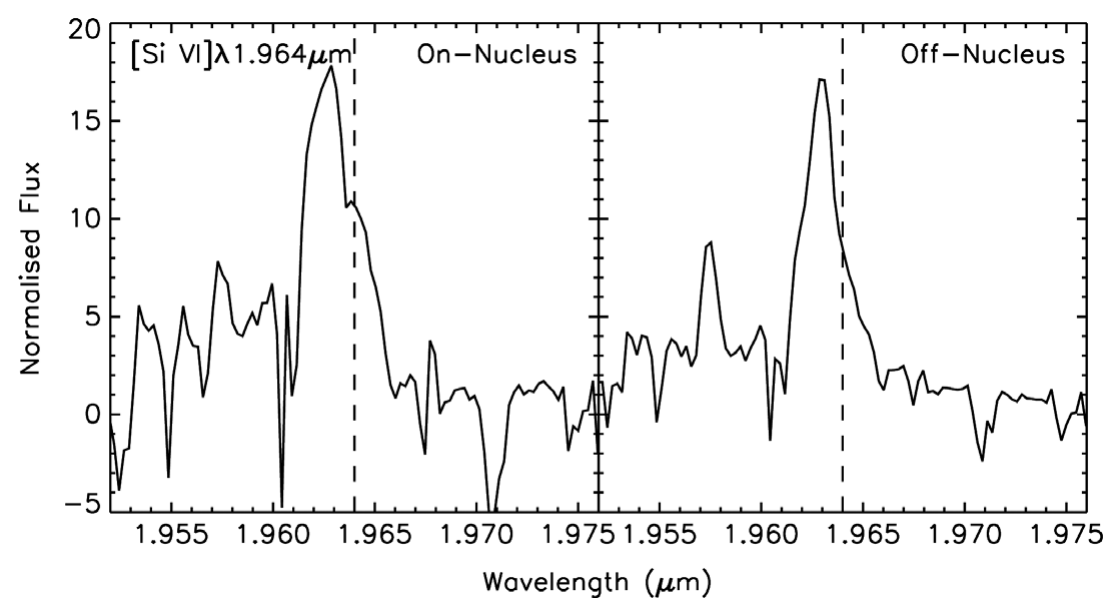

Figure 2. NGC 3783. Left: [Si vi] emission from nucleus. Right: [Si vi] off-nucleus. Dashed line shows velocity of 1-0 S(1) $\mathrm{H}_{2}$ emission line, which is also consistent with that of $\mathrm{H} \mathrm{I} 21 \mathrm{~cm}$ radio line.

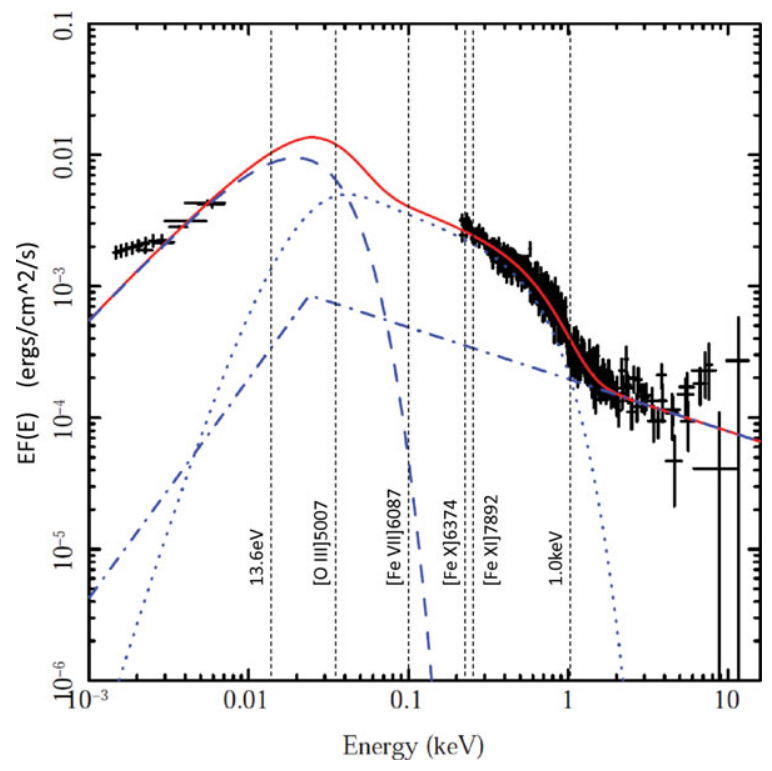

Figure 3. Example of an AGN SED fitted using three components, disk = dashed line, Compton component $=$ dotted line, and broken power-law $=$ dot-dashed line. The poor fit at lowest energies is due to stellar contamination.

extent is $\sim 42$ pc. Interestingly, the blueshift from systemic velocity of both the on and off-nucleus profile is about the same, $\sim 250 \mathrm{~km} \mathrm{~s}^{-1}$ (Figure 2). This lack of a velocity gradient is what might be expected if one were viewing a conical outflow roughly aligned along the line of sight. More detailed modeling of spatially extended emission-line ratios is in progress.

\section{Future Observational Tests}

Because the HIL emitting region is very probably statified, good spectral resolution is essential to deconvolve the line profiles. Combining this with the high spatial resolution 

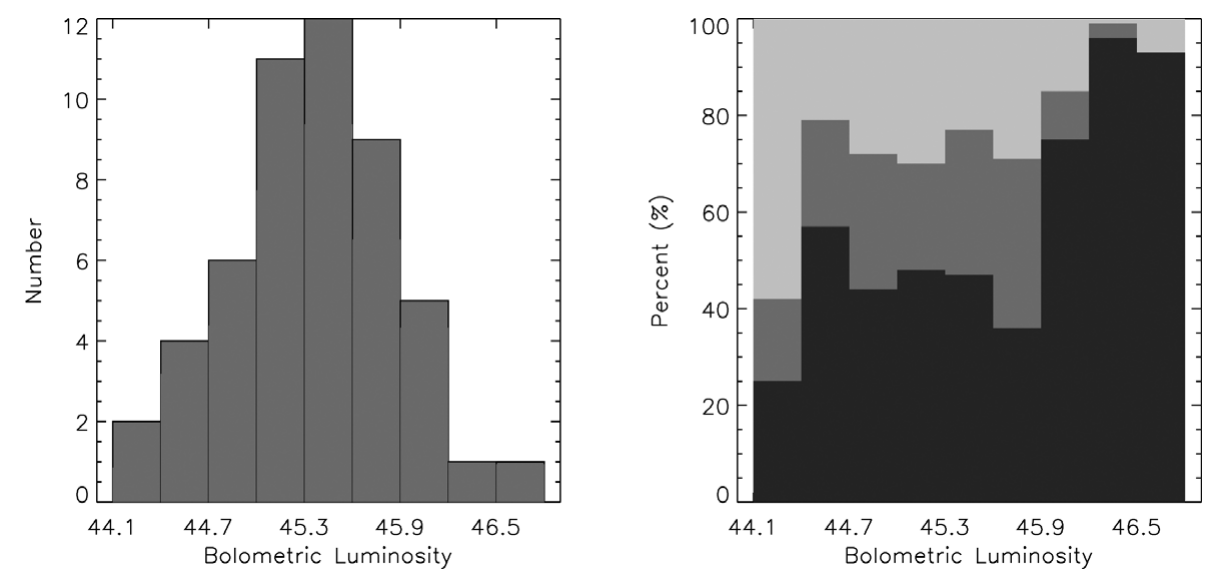

Figure 4. Histograms of a new X-ray sample of 51 AGN. Left: bolometric luminosity. Right: percentage contributed by each SED component, black = disk, dark grey = Compton component, grey $($ top $)=$ power-law.

that is now possible using IFUs such as SINFONI, a start has been made on a few nearby AGN. Other routes are also available. For example, one of the key open questions regarding the innermost HIL region is the nature of the ionization mechanism. This is assumed to be via photionization based on estimates of the gas temperature and good correlations with the high-energy continuum. However, the crucial continuum photon energies lie in the unobservable region of the spectrum. To circumvent this, we are analyzing a sample of 51 AGN, all with excellent X-ray data plus SDSS spectra. We have fitted their SEDs using three principal components; an accretion disk, a Compton component, and a broken power-law (see Figure 3 for one example). We shall be able to use the integrated luminosities of each continuum component (Figure 4) to see which correlates best with the HILs. Using black hole mass estimates from the Balmer-line profiles and continua, we shall see whether there is any relationship between the Eddington ratio and the strength, velocity width, and blueshift of the HILs.

\section{References}

Ferguson, J., Korista, K., \& Ferland, G. 1997, ApJS, 110, 287

Gelbord, J., Mullaney, J. R., \& Ward, M. 2009, MNRAS, 397, 172

Landt, H., Bentz, M., Ward, M., Elvis, M., Peterson, B. M., Korista, K., \& Karovska, M. 2008, ApJS, 174, 282

Marconi, A., van der Werf, P., Moorwood, A., \& Oliva, E. 1996, A\&̈A, 315, 335

Mazzalay, X., Rodriguez-Ardila, A., \& Komossa, S. 2010, MNRAS, in press [arXiv:1002.3152]

Mullaney, J. R. \& Ward, M. 2008, MNRAS, 385, 53

Mullaney, J., Ward, M., Done, C., Ferland, G., \& Schurch, N. 2010, MNRAS, 394, L16

Pelat, D., Alloin, D., \& Fosbury, R. 1981, MNRAS, 195, 787

Penston, M., Fosbury, R., Boksenberg, A., Ward, M., \& Wilson, A. 1984, MNRAS, 208, 364

Prieto, M. A., Marco, O., \& Gallimore, J. 2005, MNRAS, 364, L28

Reeves, J., O'Brien, P., Braito, V., Behar, E., Miller, L., Turner, T., Fabian, A., Kaspi, S., Mushotzky, R., \& Ward, M. 2009, ApJ, 701, 493

Riffel, R., Rodriquez-Ardilla, A., \& Pastoriza, M. 2006, A\& A, 457, 61

Rodriguez-Ardila, A., Prieto, M., Viegas, S., \& Gruenwald, R. 2006, ApJ, 653, 1098 